\title{
The effects of unlabeled and labeled picture cues on very young children's memory for location
}

\author{
ROBERT BLAIR, MARION PERLMUTTER, and NANCY ANGRIST MYERS \\ University of Massachusetts, Amherst, Massachusetts 01003
}

Twenty children at each of three age levels were tested in a nine-choice delayed-response task to evaluate their use of picture cues. On some trials, no picture cues were available; on others, unlabeled pictures or labeled pictures were provided. Although performance improved with age, all age groups showed more errorless trials as more cues were provided. The results suggest that children under 3 years can use picture cues, and that age-related increases in verbal label production are not responsible for the developmental improvement.

By the time children reach 18 months of age, they can store and recognize stimulus information, have developed an object concept, and are able to coordinate these accomplishments to solve simple localization problems. Over the next 2 years localization improves further. While the nature of this development is not entirely clear, both Babska (1965) and Loughlin and Daehler (1973) have suggested that only children over 3 years of age are likely to encode and use any discriminative information other than location to guide search for absent objects.

Babska's argument was based on her finding that 27-month-olds performed poorly when required to find a hidden toy in the absence of location cues. She presented a single box to the child, one of four boxes with distinctive patterns on their lids, and hid a toy in it; she then placed the box in an array with the other three boxes before the child searched. Loughlin and Daehler (1973) also found picture cues were not facilitating to children younger than 3 years, although they were helpful to older children. They examined 27-, 32-, and 42-month-olds in a four-choice delayed-response task. On one half of the trials no pictures were placed on the four stationary boxes, while on the remaining trials pictures were available. Only the oldest group demonstrated superior performance on trials in which picture cues were available.

In light of the considerable object knowledge and recognition prowess of even much younger children, these results are somewhat surprising; it is unclear why

This research was supported by NICHHD Grant HD09346 and by grants from the Spencer and Grant Foundations. It is based in part on an honors' thesis submitted by Robert Blair in partial fulfillment of requirements for an honors' BS degree at the University of Massachusetts. Marion Perlmutter is now at the Institute of Child Development, University of Minnesota. We thank the Director of the Amherst Nursery and all children and parents for cooperating in our study, and Marvin Daehler and Hilary Horn for many patient readings of previous versions of this manuscript. Requests for reprints should be sent to Nancy Myers, Department of Psychology, University of Massachusetts, Amherst, Massachusetts 01003. children under 3 years did not use the appropriate cues. The present study was designed to assess further young children's use of pictorial information in locating hidden objects and to evaluate one possible reason for their failure to profit from added picture cues, the possibility that verbal discriminative cues are necessary for effective use of pictures. If such cues are needed, labeling should improve performance. Furthermore, if a primary difference between 2- and 3-year-olds is their probability of producing verbal labels, then labeling should be more helpful to the younger children.

\section{METHOD}

\section{Design}

The experimental design was a mixed 3 (age) by 3 (cue condition) by 8 (trial blocks) design. Each child was given eight blocks of three randomly-ordered trials which included one trial of each cue condition. A different random order of presentation of cue conditions within trial blocks was used for each subject within an age group.

\section{Subjects}

Twenty children at each of three age levels served as subjects. The three age levels included 10 boys and 10 girls averaging 27 months of age (26 to 29 months), 10 boys and 10 girls averaging 33 months ( 31 to 36 months), and 9 boys and 11 girls averaging 45 months (40 to 52 months). An additional male in the youngest group and two males in the middle group, unwilling to complete the entire task, were dropped from the experiment. Children in the two youngest groups participated in the Child Behavior Laboratory at the University of Massachusetts. Children in the oldest group were students in the Amherst Nursery, and were tested in a research trailer at their school.

\section{Apparatus and Materials}

A nine-drawer IBM card-file cabinet was used as the apparatus. It was $61 \times 40 \times 30 \mathrm{~cm}$, with drawers $19 \times 10 \mathrm{~cm}$. The nine drawers were arranged in a 3 by 3 array, and the entire apparatus was located atop a child-sized table in front of the subject's chair. Another smaller table was located behind the subject, and held a balance toy on which rings could be stacked during delay periods. A Sony tape recorder was used to signal the end of delay periods.

Two sets of nine $8 \times 8 \mathrm{~cm}$ colored picture cards were used as picture cues. They were laminated in clear pliofilm, and had small magnets mounted on their backs to permit attaching them 
to the front of the drawers. The pictures were of objects shown in the Peabody Picture Vocabulary Test at the 2-year-old age level. One set consisted of pictures of an apple, broom, cat, chair, hand, key, shoe, spoon, and truck, and the other of a bus, cup, dog, flag, knife, pie, sweater, table, and tree. Small crackers were used as hidden objects.

\section{Procedure}

All subjects were tested individually. After becoming acquainted with the experimenter in a playroom, the subject was invited to play a game in a nearby experimental room. A parent of each child in the two younger groups was usually present and was seated behind the table.

The experimental session began with three practice trials, which served to familiarize the child with the procedure and to provide an example of each of the three cue conditions. The procedure for each cue condition follows.

No-picture condition. The experimenter directed the child's attention to the drawer where the object was being hidden and, while closing the drawer, said "I'm hiding it here."

Unlabeled-picture condition. The experimenter placed one set of pictures on the fronts of the drawers, directed the child's attention to the drawer where the object was being hidden, and, while closing the drawer, pointed to the picture and said "I am hiding it here."

Labeled-picture condition. The experimenter placed the other set of pictures on the fronts of the drawers, directed the child's attention to the drawer where the object was being hidden, and, while closing the drawer, pointed to the picture and verbally labeled it. For example, the experimenter might say "I am hiding it here with the cat."

Practice trials employed no delay period. The subject observed the object being hidden and was immediately instructed to search for it. If the child chose the correct location, he was verbally reinforced and allowed to eat the cracker or save it in a paper bag to take home. If the child's choice was incorrect, he was encouraged to search until correct or until four errors were made, in which case the experimenter showed the child the correct location of the hidden object. All subjects experienced the same order of cue conditions for practice trials. On the first practice trial, labeled pictures were provided; on the second, unlabeled pictures were used; and on the third, no pictures were provided.

The 24 test trials were administered in essentially the same manner as the practice trials, except that a $25-\mathrm{sec}$ delay period was employed. After the object was hidden, the child was turned $180 \mathrm{deg}$ and encouraged to play with the balance toy. When a short tone signaled the end of the delay period, the child was turned back toward the apparatus and instructed to search for the hidden object.

Different random sequences of locations for hiding objects were used for each subject in an age group. The same position was not used on any two consecutive trials, and the object was hidden in every position except the center in each of the cue conditions; thus, in 24 trials, each child received 8 trials in each cue condition, and the object was hidden in a different position for each trial of a condition. Each set of pictures was used in the unlabeled-picture cue condition for half the subjects in each age group and in the labeled-picture cue condition for the remaining subjects. Thus, a different picture set was employed for each cue condition in which picture cues were available, and the object was hidden once with each picture for every subject.

\section{RESULTS}

\section{Percentage Errorless Trials}

The mean percentage of errorless trials in each cue condition for the three age groups is shown in Table 1 .
Table 1

Mean Percentage of Errorless Trials

\begin{tabular}{llllc}
\hline & \multicolumn{3}{c}{ Cue Conditions } & \multirow{2}{c}{$\begin{array}{c}\text { Combined } \\
\text { Over }\end{array}$} \\
\cline { 2 - 4 } Age Groups & NP & UP & LP & Conditions \\
\hline 27 Months & 50 & 66 & 68 & 61 \\
33 Months & 52 & 67 & 80 & 66 \\
45 Months & 55 & 81 & 91 & 76 \\
Combined Over Ages & 52 & 71 & 80 & \\
\hline
\end{tabular}

Note $-N P=$ no picture; $U P=$ unlabeled picture; $L P=$ labeled picture.

All age groups responded above chance level under all cue conditions, and in each condition performance was ordered according to age: 45 -month-olds did better than 33-month-olds, who did better than 27-month-olds. The mean percentage of errorless trials for the oldest, middle, and youngest groups, respectively, was $76 \%, 66 \%$, and $61 \%$. An analysis of variance on these data revealed a significant age main effect $[\mathrm{F}(2,57)=5.23, \mathrm{p}<.01]$. Bonferoni $t$ tests were carried out to compare performances of each of the age groups. The tests indicated significantly more errorless trials for the oldest group than for the youngest group (EW $<.01)$, but the percentage of errorless trials for the middle group was not significantly different from either of the other groups.

As may be seen in Table 1, the percentage of errorless trials increased as more cues were provided. The mean percentage of errorless trials for the no-picture, unlabeled-picture, and labeled-picture cue conditions, respectively, was $52 \%, 71 \%$, and $80 \%$. The cue condition main effect was significant $[F(2,57)=54.44$, $\mathrm{p}<.001]$, and planned comparisons indicated that performance under each of the three cue conditions was significantly different from the others (all EWs $<.01$ ).

All age groups were similarly affected by cue conditions; the Age by Cue Condition interaction failed to reach statistical significance $[F(4,114)=2.26, p>.05]$. Performance did not change over the experimental session; trial blocks was not a significant source of variance.

\section{Error Analyses}

Analyses were carried out to determine if subjects were selecting any of the nine locations at above-chance level or if preferences for specific rows or columns were evidenced. None of the nine locations, three rows, or three columns was selected at above-chance level by any age group. Analyses also indicated that each age group demonstrated a tendency to return to the location correct on the preceding trial at above-chance level $(\mathrm{p}<.05)$.

\section{DISCUSSION}

Although this task involved nine choices, rather than the four previously employed, the results once again indicate considerable competency of 2- and 3-year-olds in remembering and finding 
hidden objects and improvement over the age range. Furthermore, in direct contrast to the Loughlin and Daehler (1973) data, even the youngest children performed better on trials in which picture cues were available. One reason for the disparity in the results of the two studies is suggested by the additional finding of the present investigation: that performance was best on trials in which labeled pictures were provided. It is possible that the experimenter providing labels in the condition introduced in this study encouraged children at all three ages to produce and utilize their own labels in the unlabeled-picture condition.

Under some circumstances, then, even children under 3 years make use of discriminative cues to facilitate memory and search for the location of hidden objects. The better performance of all children in the labeled-picture condition indicates, however, that verbal labels do enhance the effects of picture cues and that the children were probably not consistently effective in spontaneous verbal label production in the unlabeled-picture condition. Moreover, our hypothesis that experimenter-provided labels might be more helpful to the younger children was not confirmed. The developmental improvement in performance could not be attributed, then, to an age-related increase in spontaneous verbal label production. This is consistent with Perlmutter and Myers' (1976) finding that spontaneous labeling does not change over the preschool years and with their suggestion that mechanisms other than verbal label production account for age-related improvement in recognition during this time period.
One additional finding is worth noting. An error analysis revealed that all age groups tended to search the location correct on the preceding trial more often than expected by chance. This behavior is characteristic of children in Piaget's Stage IV. It is interesting that, although their predominant search strategy is to the correct location, even children almost 4 years of age occasionally show the tendency to search for a hidden object in the location where it was previously found.

\section{REFERENCES}

BABSKA, Z. The formation of the conception of identity of visual characteristics of objects seen successively. In P. Mussen (Ed.), European research in cognitive development. Monographs of the Society for Research in Child Development, 1965, 30(2. Serial No. 100), 112-124.

Loughlin, K. A., \& DAEHLER, M. W. The effects of distraction and added perceptual cues on the delayed reaction of very young children. Child Development, 1973, 44, 384-388.

Perlmutter, M., \& Myers, N. A. Recognition memory development in preschool children. Developmental Psychology, 1976, 12 271-272.

(Received for publication October 3, 1977.) 